\title{
Organischer Staub am Arbeitsplatz: Messung der pyrogenen Aktivität
}

\author{
Organic Dust at Workplaces: Measurement of Pyrogenic Activity
}

Autoren

Institut
V. Liebers, S. Brinkert, H. Stubel, T. Brüning, M. Raulf-Heimsoth

Institut für Prävention und Arbeitsmedizin der Deutschen Gesetzlichen Unfallversicherung, Institut der Ruhr-Universität Bochum (IPA) (Prof. Dr. Thomas Brüning) eingereicht 18.3.2010 akzeptiert nach Revision 16. 4. 2010

\section{Bibliografie}

Dol http://dx.doi.org/ 10.1055/s-0029-1244171

Online-Publikation: 8. 6. 2010

Pneumologie 2010; 64:

619-625 @ Georg Thieme

Verlag KG Stuttgart · New York ISSN 0934-8387

\section{Korrespondenzadresse}

Dr. Verena Liebers

Institut für Prävention und Arbeitsmedizin der Deutschen Gesetzlichen Unfallversicherung Institut der Ruhr-Universität Bochum (IPA)

Bürkle-de-la-Camp-Platz 1

44789 Bochum

liebers@ipa-dguv.de

\section{Zusammenfassung \\ $\nabla$}

Hintergrund: Organischer Staub kann in seiner Zusammensetzung sehr komplex sein. Für exponierte Personen sind gesundheitliche Belastungen möglich. An entsprechenden Arbeitsplätzen, u.a. in der Land- und Abfallwirtschaft, kann es deshalb zu Atemwegsbeschwerden kommen. Messmethoden, die die Zusammensetzung und Aktivität der Staubkomponenten bestimmen, sind deshalb wichtig. Sie ermöglichen eine Risikoabschätzung und können Interventionsmaßnahmen begleiten. Ziel der vorliegenden Studie war die Etablierung des Vollbluttests (VBT), basierend auf kryo-konserviertem Blut, um organische Staubproben hinsichtlich ihrer pyrogenen Aktivität zu charakterisieren.

Methode: 30 Filterstaubproben verschiedener Arbeitsplätze sowie einzelne Modellsubstanzen (E. coli Endotoxin, Zymosan, Aspergillus versicolor-Extrakt) wurden mittels VBT, basierend auf kryo-konserviertem Blut, analysiert. Zusätzlich wurden die Arbeitsplatzproben im Limulus-Amöbozyten-Lysat-Test (LAL-Test) hinsichtlich Endotoxin-Aktivität untersucht. Der VBT ist ein Zweistufentest, humanes Vollblut wird mit einem Stimulus inkubiert und anschließend die Mediatorenfreisetzung (u. a. IL-1 $\beta$, monocyte chemotactic protein 1 = MCP-1) im Überstand gemessen.

Ergebnisse: Mit Hilfe des VBT konnte die pyrogene Aktivität von 30 Arbeitsplatz-Staubproben beschrieben werden. Die IL-1 $\beta$-Freisetzung der Proben aus kryo-konserviertem Blut lag zwischen 33 und 500000 pg $/ \mathrm{ml}$. Die Korrelation der Ergebnisse zum LAL-Test betrug $\mathrm{r}^{2}=0,9$ (Pearson, $p=5,0 E-07)$. Als weiterer Messparameter neben IL-1 $\beta$ wurde MCP-1 eingesetzt. Immunologisch aktive Substanzen wie A. versicolor-Extrakt, die keine IL-1 $\beta$-Aktivität im Testsystem auslösen, konnten durch den MCP-1-Nachweis erfasst werden.

\section{Abstract \\ $\nabla$}

Background: Organic dust is composed of numerous components. Exposed people may have health problems. Respiratory tract complaints may occur at workplaces like those in agriculture or in the waste industry. Therefore methods that determine the composition and activity of dust components are important. They help in the performance of risk estimations and could accompany intervention studies. The aim of our study was to establish a whole blood assay using cryo-preserved blood for characterisation of the pyrogenic activity originating from organic dust samples.

Methods: 30 dust filter extracts from several workplaces as well as E. coli endotoxin, zymosan and Aspergillus versicolor extract were analysed using the whole blood assay. In addition, all workplace samples were analysed by means of the Limulus amoebocyte lysate test (LAL test) with regard to endotoxin activity. The whole blood assay is a two-step assay: Firstly, blood is incubated with the stimulus and, secondly, mediators released in the supernatant were determined (IL1$\beta$, MCP-1)

Results: It was possible to detext pyrogenic activity for all 30 dust filter extracts. IL- $1 \beta$ release from cryo-preserved blood was in the range of 33 to $500000 \mathrm{pg} / \mathrm{mL}$. Correlation with the results of the LAL test was $r=0.9(p=5,0 E-07)$. In addition to IL-1 $\beta$, measurement of MCP-1 was undertaken. Immunological activity of substances like Aspergillus versicolor extract, which induced no IL-1 $\beta$ release, could be detected via determination of MCP-1.

Conclusions: Cryo-preserved blood offers the possibility to quantify pyrogenic activity of dust samples by measuring IL-1 $\beta$ and MCP-1. The whole blood assay can be used as a standardised method for description of the dust load at workplaces. 
Schlussfolgerungen: Kryo-konserviertes Blut ist geeignet, um den VBT durchzuführen. Im Gegensatz zu Frischblut ermöglicht es, Belastungen unabhängig von der individuellen Beanspruchung zu beschreiben. Die pyrogene Aktivität arbeitsplatzbezogener Staubextrakte kann quantifiziert werden, indem die IL$1 \beta$ - oder MCP-1-Freisetzung des Bluts nach Stimulation mit den zu prüfenden Substanzen ermittelt wird.

\section{Einleitung}

$\nabla$

An zahlreichen Arbeitsplätzen, wie z.B. in der Landwirtschaft, der Abfallwirtschaft oder der baumwollverarbeitenden Textilindustrie, können Bioaerosole in schädigenden Konzentrationen auftreten [1]. Vor allem Bereiche mit hoher Staubentwicklung müssen deshalb entsprechend überwacht werden. Je nach Inhaltsstoffen können organische Stäube infektiös, toxisch, allergisierend, reizend oder belästigend wirken, wobei ihre qualitative und quantitative Zusammensetzung komplex ist. Zu den Komponenten organischer Stäube gehören Bakterienbestandteile wie Endotoxine (Lipopolysaccharide = LPS) und andere fieberauslösende (pyrogene) Substanzen, die über die Atemwege aufgenommen werden können [2-4]. Das Immunsystem hat verschiedene Möglichkeiten, um auf mikrobielle Komponenten zu reagieren (siehe $\bullet$ Abb. 1).

Wichtiger Bestandteile der Immunabwehr sind die so genannten Mustererkennungsrezeptoren ( $P R R=$ pattern recognition receptor), wie z.B. die „Toll-Like-Rezeptoren“ (TLR). Sie erkennen molekulare Muster (PAMPs = pathogen associated molecular patterns), die sich auf Krankheitserregern befinden. TLR sind Transmembranmoleküle, die selektiv bakterielle Komponenten wie z.B. LPS binden. Die LPS-Bindung führt zur Aktivierung verschiedener Proteine und Enzyme und des Transkriptionsfaktors NFKB. Er steuert die Proteinbiosynthese diverser Gene, vor allem die von proinflammatorischen Mediatoren. Zytokine wie Interleukin-1 $\beta$ (IL-1 $\beta$ ), Tumornekrosefaktor $\alpha$ (TNF- $\alpha$ ) oder IL-6 werden ausgeschüttet, locken weitere immunkompetente Zellen an und bewirken letztlich die Vernichtung bzw. Neutralisierung der mikrobiellen Komponenten.

Bisher sind zehn Mitglieder der TLR-Familie genauer charakterisiert (TLR 1 -10). Im Hinblick auf bakterielle Proteine spielen vor allem TLR-2 (für Lipoprotein) und TLR-4 (für LPS) eine wichtige Rolle. Aber auch andere Rezeptoren sind an der Abwehr beteiligt. Eine Schlüsselposition bei Pilzinfektionen kommt dem Dectin-1 zu. Dieser Lektin-ähnliche Rezeptor erkennt $\beta$-Glucane und kann sowohl eine eigene Signalkaskade anstoßen als auch im Zusammenwirken mit den TLR zu einer Immunantwort führen [5]. Verschiedene Forschungsergebnisse weisen darauf hin, dass die Stimulation von TLR oft zeitgleich mit anderen Oberflächenmolekülen, z.B. dem IgE-Rezeptor, geschieht. Damit kommt es zu einer Verschränkung der angeborenen und der erworbenen Immunantwort [6].

Das Vorhandensein mikrobieller Komponenten ist nicht grundsätzlich gefährlich, sondern ein normaler Bestandteil der Umwelt. Sogar positive Effekte wurden im Zusammenhang mit Endotoxin-Exposition in epidemiologischen Studien beschrieben: ein verringertes Auftreten von Allergien [7,8] und Krebserkrankungen [9]. Wie sich die Inhalation von organischem Feinstaub auswirkt, hängt dabei sowohl von der Dosis als auch vom Wirkort, dem Zeitpunkt der Exposition und der persönlichen Empfänglichkeit ab.

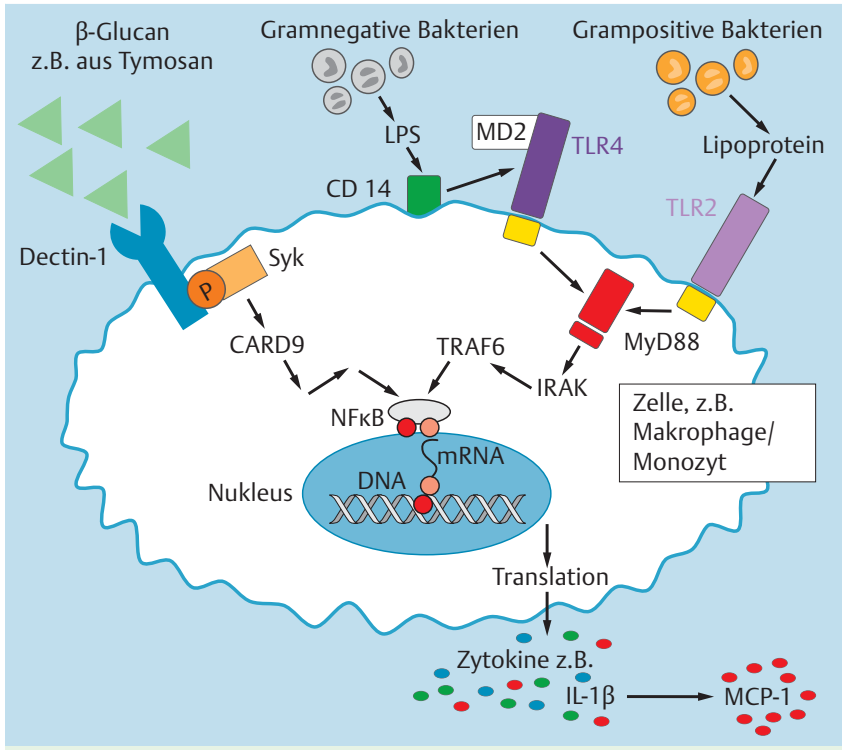

Abb. 1 Schematische Darstellung der Immunantwort auf mikrobielle Substanzen. Unter Beteiligung verschiedener Rezeptoren kommt es zu einer Signalkaskade und letztlich zur Aktivierung des Transkriptionsfaktors NFkB und damit zur Freisetzung proinflammatorischer Botenstoffe. CARD = caspase recruitment domain (Protein); SYK = spleen tyrosine kinase; LPS = lipopolysaccharid; TLR = Toll-like receptor; MyD88 = myeloid differentiation protein (adapter protein for TLR); NFKB = transcription factor; IRAK = serin kinase; TRAF 6 = tumor-necrosis factor receptor associated factor 6 (adapter protein); MD 2 = accessory protein of TLR; MCP-1 = monocyte chemotactic protein 1 , nach neuer Nomenklatur CCL-2 = chemokine (C-C-motif) ligand 2; IL-1 $\beta=$ Interleukin-1 $\beta$.

Unbestritten ist jedoch, dass es im Rahmen von hohen Endotoxin-Expositionen zu Beschwerden in den Atemwegen kommen kann [10]. Als Akuteffekt ist die toxische Alveolitis (=Organic Dust Toxic Syndrome, ODTS) bekannt, eine systemische Entzündungsreaktion mit grippeähnlichen Symptomen. Chronische Effekte können sich in Form von Atembeschwerden, Fieber, Brustenge u.ä. äußern.

Daher ist es wichtig, dass Methoden zur Analyse von Bioaerosolen zur Verfügung stehen. Eine Möglichkeit, die mikrobiellen Komponenten zu analysieren, ist es, die Gesamtkeimzahlen in Form Kolonie-bildender Einheiten (KBE) quantitativ zu erfassen. Da Endotoxin allerdings auch aus abgestorbenen Bakterien stammen kann, wird vor allem der sogenannte LAL-Test (LimulusAmoebozyten Lysat Test) zur Endotoxin-Bestimmung eingesetzt. Dafür verwendet man die Hämolymphe des Pfeilschwanzkrebses, die bei Kontakt mit Endotoxin gerinnt. Der LAL-Test ist international etabliert und liefert - standardisiertes Vorgehen vorausgesetzt - vertrauenswürdige Ergebnisse zur Abschätzung der Endotoxin-Aktivität [11-13]. Gegenüber Nicht-Endotoxin-Bestandteilen, wie sie in Bioaerosolen regelhaft vorkommen, ist der LAL-Test störanfällig [14]. LAL-Test und Gesamtkeimzahlbestimmung bilden allerdings nur den bakteriellen Anteil der Bioaerosole (partiell) ab.

Um der komplexen Zusammensetzung von Bioaerosolen gerecht zu werden, sind also weitere Methoden erforderlich. Eine Möglichkeit ist der Vollbluttest (VBT): Hier wird die Fähigkeit der Staubkomponenten zur Induktion von pyrogener Aktivität überprüft. Zu diesem Zweck wird humanes Vollblut mit dem Staubextrakt inkubiert und anschließend werden im zellfreien Überstand proinflammatorische Zytokine wie z.B. IL-1ß nachgewiesen. 
In unserer Studie wurde der VBT mit kryo-konserviertem Blut für arbeitsplatzbezogene Filterstaubproben validiert [2], wobei im Gegensatz zu Kindinger et al. [15] nicht die kompletten Staubfilter, sondern Filterextrakte verwendet wurden. Kindinger et al. arbeiten mit speziellen Filterkartuschen, in die zuerst der Staub gesammelt wird und anschließend direkt das Blut gefüllt wird. Dadurch ist ein Filter mit einer Messung verbraucht. Durch die Extraktion der Staubfilter mit Wasser entsteht dagegen ein Extrakt, der für mehrere Messungen eingesetzt werden kann. Folglich ist z.B. die Validierung mittels Doppelbestimmung möglich, aber vor allem können die Proben in unterschiedlichen Verfahren analysiert werden. In der vorliegenden Studie wurden die Proben sowohl im LAL-Test als auch im VBT untersucht. Mit Hilfe dieser beiden Verfahren soll ein Analyseschema entwickelt werden, dass zur vergleichenden Charakterisierung von Staubproben eingesetzt werden kann. Zur Validierung der Tests kamen neben den Staubextrakten auch E. coli, Zymosan und Aspergillus versicolor als Reinsubstanzen zum Einsatz. E. coli ist ein ubiquitäres Bakterium, Zymosan Bestandteil eines Hefepilzes und Aspergillis versicolor ein Pilz, der typischerweise bei Feuchteschäden in Innenräumen auftritt [16] und zu gesundheitlichen Problemen führen kann.

Langfristiges Ziel ist es, die Bioaerosol-Exposition an Arbeitsplätzen mit Hilfe unterschiedlicher, sich ergänzender Verfahren zu beurteilen.

\section{Material und Methoden}

\section{Staubsammlung und Filterextraktion}

Die luftgetragenen Staubfilterproben stammten aus der Getreideverarbeitung und -lagerung $(n=20)$, aus Hühner- und Taubenställen $(n=2)$ sowie einer Ferkelzucht $(n=2)$. Sammlung und Extraktion wurden nach dem bereits beschriebenen Protokoll durchgeführt [1]. Die Filterextraktion erfolgte in Borosilikat Erlenmeyer-Kolben, indem die Filter auf einem Horizontalschüttler (130 Schüttelbewegungen/Min.) in 5 bzw. $10 \mathrm{ml}$ pyrogenfreiem Wasser (Braun, Melsungen, Deutschland) 60 Min. bei Raumtemperatur geschüttelt wurden. Anschließend wurde die Flüssigkeit in ein Reagenzglas überführt, zentrifugiert (1000 g/10 Min.), aliquotiert und bei $-80^{\circ} \mathrm{C}$ gelagert.

\section{Limulus-Amöbozyten-Lysat (LAL)-Test}

Die Endotoxin-Bestimmung erfolgte mittels Chromo LAL-Test (Haemochrom Diagnostica, Essen, Germany, Kontrollstandard E. coli 0113:H10). Die Endotoxin-Aktivität wurde in EU/ml angegeben. Jeweils $100 \mu$ l des aufgetauten Extrakts inkubierten $10 \mathrm{Min}$. lang bei $37^{\circ} \mathrm{C}$ in einer Mikrotiterplatte (96 Vertiefungen, Becton Dickinson, Heidelberg). Anschließend erfolgte die Zugabe von LAL-Reagenz (in pyrogenfreiem Wasser gelöst) und Kinetik wurde mit einem Mikroplattenreader (Spectra MAX 340 PC, Software Softmax 3 [Molecular Devices, Sunnyvale, USA]) bei $405 \mathrm{~nm}$ und $37^{\circ} \mathrm{C}$ erfasst. $\mathrm{Zu}$ jeder Messung gehörte eine frische Standardkurve im Bereich 0,005 bis $50 \mathrm{EU} / \mathrm{ml}$. Die Staubproben kamen in $1: 10$ oder $1: 100$ Verdünnung zum Einsatz. Um die Messgenauigkeit zu belegen, wurde für jede Probe die Wiederfindungsrate von $5 \mathrm{EU} / \mathrm{ml}$ Endotoxin (Kontrollstandard) geprüft, die im Bereich von 50 bis $200 \%$ liegen musste („spike-Probe“). Als Negativ-Kontrolle diente pyrogenfreies Wasser.

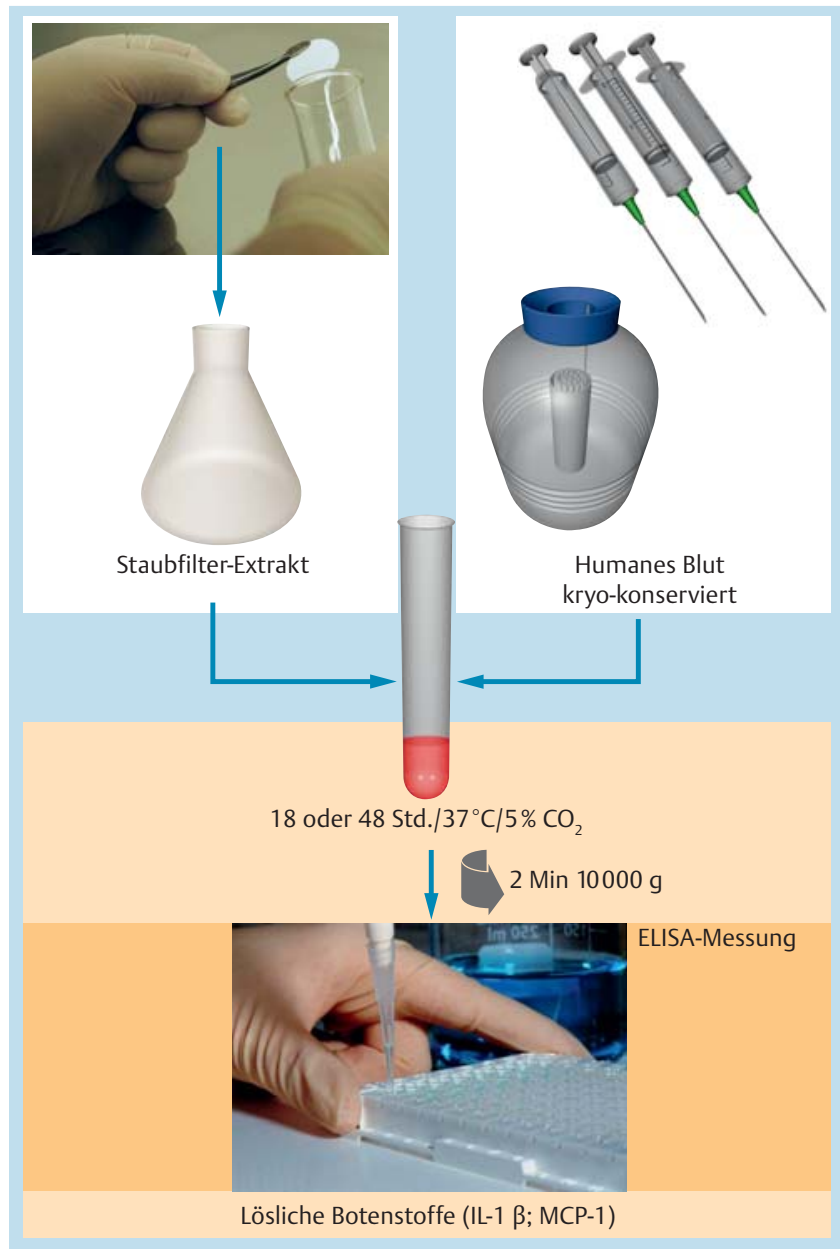

Abb. 2 Schematischer Ablauf des Vollbluttests (VBT). Im zellfreien Überstand werden mittels ELISA lösliche Botenstoffe nachgewiesen, die Blutzellen nach Stimulation mit dem Staubextrakt freigesetzt haben.

\section{Vollbluttest}

Der VBT ist ein Zweistufentest ( Abb. 2).

Im ersten Schritt wird humanes Vollblut mit der zu untersuchenden Probe für 18 oder 48 Stunden inkubiert. Durch die Zellaktivierung werden Zytokine freigesetzt, die in einem zweiten Schritt nach Zentrifugation im zellfreien Überstand mittels spezifischen ELISAs nachgewiesen werden.

\section{a) Stimulation von kryo-konserviertem Blut}

Kryo-konserviertes Blut (Mischung aus fünf zufällig ausgewählten Spendern, Zwisler Laboratorium, Konstanz, Deutschland) wurde aufgetaut und sofort in Zellkulturmedium RPMI-1640 verdünnt (1,5 ml Kryo-Blut plus $6 \mathrm{ml}$ RPMI, vorgewärmt auf $37^{\circ} \mathrm{C}$ ). Jeweils $500 \mu$ l des verdünnten Bluts wurden mit $100 \mu$ der Probe versetzt und $400 \mu \mathrm{l}$ RPMI zugegeben. Anschließend inkubierte das Gemisch für $18 \mathrm{Std}$. bzw. $48 \mathrm{Std}$. bei $37^{\circ} \mathrm{C}$ und $5 \% \mathrm{CO}_{2}$. Nach Zentrifugation ( 2 Min. $10000 \times \mathrm{g}$ ) wurde der zellfreie Überstand des kryo-konservierten Bluts aliquotiert und bei $-80^{\circ} \mathrm{C}$ bis zur Analyse gelagert.

Als Stimulus dienten E. coli 0113:H10, Zymosan (aus Saccharomyces cerevisiae, Sigma-Aldrich; Steinheim, Deutschland, Lot.Nr.: 142164950209026 , suspendiert in 0,9\% $\mathrm{NaCl}$ ) und Aspergillus versicolor (Allergon, Ängelholm, Schweden, batch: 101507011). Der Aspergillus-Extrakt wurde hergestellt, indem jeweils 500 mg Substanz mit $10 \mathrm{ml}$ Aqua bidest gemischt (vortex) 


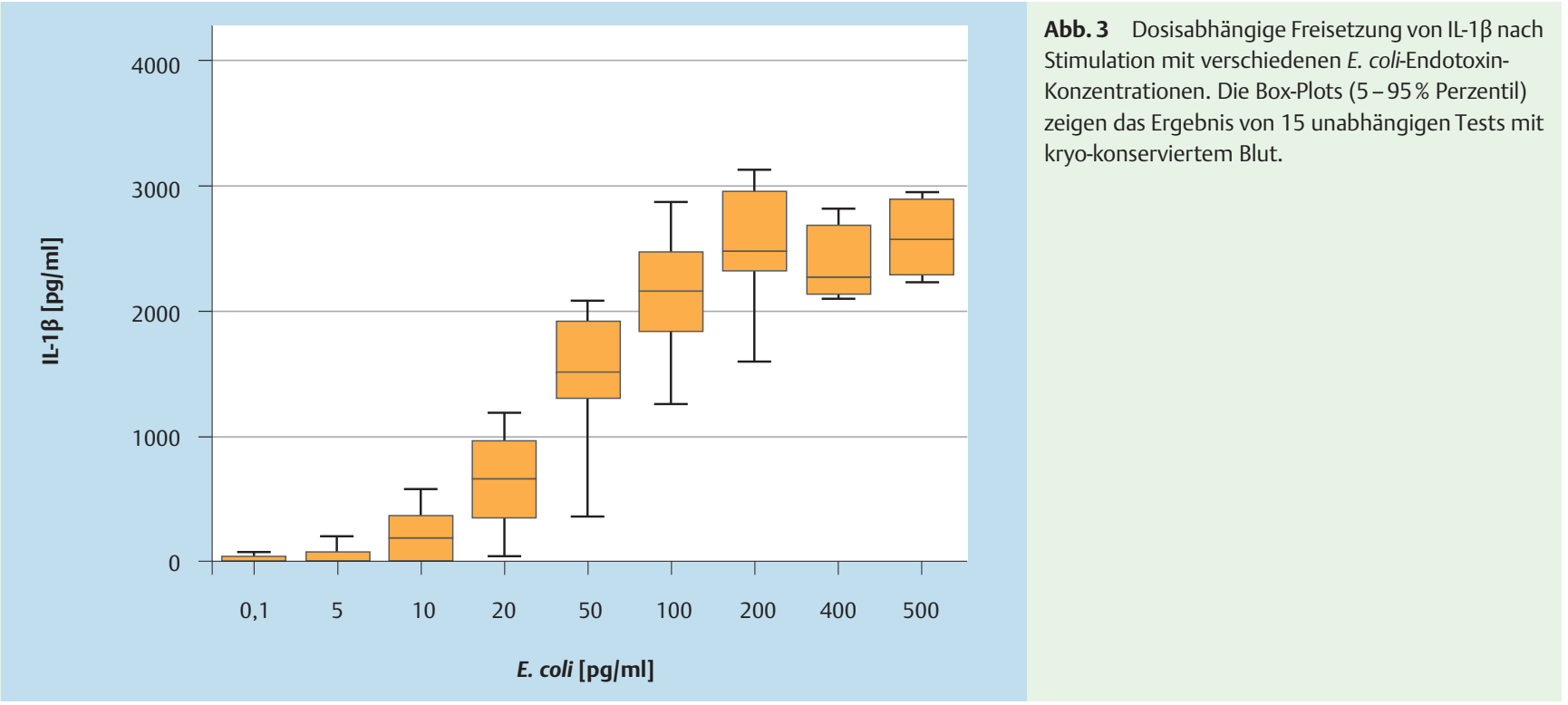

und 1 Std. geschüttelt wurde. Nachdem die Probe bei $120^{\circ} \mathrm{C}$ autoklaviert und zentrifugiert (10 Min.; $3000 \mathrm{~g}$ ) worden war, wurde der Überstand bei $-80^{\circ} \mathrm{C}$ gelagert. Vor der Verwendung im VBT wurde der Extrakt für $30 \mathrm{Min}$. auf $80^{\circ} \mathrm{C}$ erhitzt.

\section{b) Zytokinmessung}

Um die Zytokine im zellfreien Überstand zu quantifizieren, wurden IL-1 $\beta$, IL-6, TNF- $\alpha$, IL-8 und MCP- 1 mit dem monoklonalen „Sandwich“-Enzym-ELISA gemessen (IL-1 $\beta$, IL-6 und TNF- $\alpha$ : DuoSet $^{\mathrm{TM}}$ ELISA Development system; R\&D Systems, Wiesbaden Deutschland; IL-8 und MCP-1 (monocyte-chemoattractant-protein-1): Becton Dickinson). Der Messbereich lag bei 3,9-250 pg/ $\mathrm{ml}$ für IL-1 $\beta, 4,68-600$ pg/ml für IL-6, 3-200 pg/ml für IL-8, $15,6-1000 \mathrm{pg} / \mathrm{ml}$ für TNF- $\alpha$ und 7,8-500 pg/ml für MCP-1. Alle Proben wurden in zwei bis drei Verdünnungen zur Bestimmung eingesetzt. Lag der Variationskoeffizient für eine Mehrfachbestimmung über $25 \%$, wurde die Messung wiederholt.

\section{Statistische Methoden}

Um die Ergebnisse zu beschreiben, wurden Median und Mittelwert verwendet. Um die Korrelation verschiedener Variablen zu charakterisieren, wurde der Korrelationskoeffizient nach Pearson berechnet. Zusätzlich wurde der Variationskoeffizient (Verhältnis von Standardabweichung zum Mittelwert) als Streuungsmaß einer Wahrscheinlichkeitsverteilung angegeben (Programm Graphpad Prism).

\section{Ergebnisse \\ $\nabla$}

\section{IL-1 $\beta$-Freisetzung nach Stimulation von}

kryo-konserviertem Blut mit E. coli-Endotoxin

Eine 18-stündige Stimulation von kryo-konserviertem Blut mit E. coli-Endotoxin führt zu einer dosisabhängigen Freisetzung von IL-1及 ( $\bullet$ Abb. 3).

Eine lineare Zunahme der IL-1及-Antwort findet sich mit E. coliEndotoxin-Konzentrationen von 20 bis 150 pg/ml. Höhere Konzentrationen dieses Stimulus bewirken keine weitere Steigerung der IL-1 $\beta$-Freisetzung, ein Plateaubereich stellt sich bei einer IL$1 \beta$-Freisetzung von ca. $2500 \mathrm{pg} / \mathrm{ml}$ ein.
Analyse von Staubfilterextrakten mit LAL-Test und VBT

30 Staubfilterextrakte von unterschiedlichen Arbeitsplätzen wurden sowohl mit dem LAL-Test als auch mittels VBT (IL-1ßFreisetzung aus kryo-konserviertem Blut) untersucht. Für den VBT wurden die Proben so weit verdünnt, dass die induzierte IL$1 \beta$-Freisetzung innerhalb des linearen Bereichs lag, der sich nach Stimulation mit E. coli-Endotoxin ermitteln lässt. Nach Rückrechnung mit dem Verdünnungsfaktor ergibt sich für $87 \%$ der Proben (26 von 30) eine IL-1 $\beta$-Freisetzung über $2500 \mathrm{pg} / \mathrm{ml}$. Das entspricht der Zytokinfreisetzung, die durch 150 pg/ml Endotoxin ausgelöst wird.

Die Proben Nummer 1 und 2 sind im LAL-Test nahe der Nachweisgrenze, während die pyrogene Aktivität mit 33 bzw. 183 pg/ $\mathrm{ml}$ IL-1 $\beta$-Freisetzung eindeutig quantifizierbar ist.

Der mittlere Variationskoeffizient (CV) einer Wiederholungsmessung derselben Probe ergab im VBT 21\% ( $n=22$ Staubfilterextrakte) und im LAL-Test $23 \%$ ( $n=40$ Staubfilterextrakte).

- Abb. 4 zeigt die Ergebnisse der 30 Staubfilterextrakte im LALTest und im VBT. Die Korrelation der beiden Testergebnisse beträgt $r^{2}=0,9$ (Pearson).

\section{Erweiterung des VBT durch die MCP-1 Messung}

Das Standardprotokoll des VBT (IL-1 $\beta$-Freisetzung nach 18 Stunden) lässt sich erweitern, indem andere Zytokine und/oder andere Zeitpunkte gewählt werden [2].

Anhand der Extrakte von Zymosan und A. versicolor sollte getestet werden, ob die Freisetzung von MCP-1 nach 48 Stunden Stimulation ein geeigneter Parameter für den Nachweis von Pilzkomponenten ist. Da der verwendete $A$. versicolor-Extrakt keine IL-1 $\beta$-Freisetzung induzierte, ist das ein Hinweis, dass die pyrogene Aktivität von Pilzen und ihren Komponenten nur über andere Parameter bestimmt werden kann.

Die optimale MCP-1-Freisetzung ist nach $48 \mathrm{Std}$. Inkubation messbar. Abb. 5 zeigt den Vergleich der Reaktion von E. coli, Zymosan und A. versicolor hinsichtlich MCP-1-Freisetzung.

Nach einem linearen Anstieg der MCP-1-Freisetzung wird ein Maximum erreicht, höhere Stimulus-Konzentrationen bewirken wiederum eine lineare Abnahme (biphasischer Verlauf). Um eine MCP-1-Antwort zu messen, muss die Probe also in einem eng umschriebenen Konzentrationsbereich vorliegen: Eine Kon- 


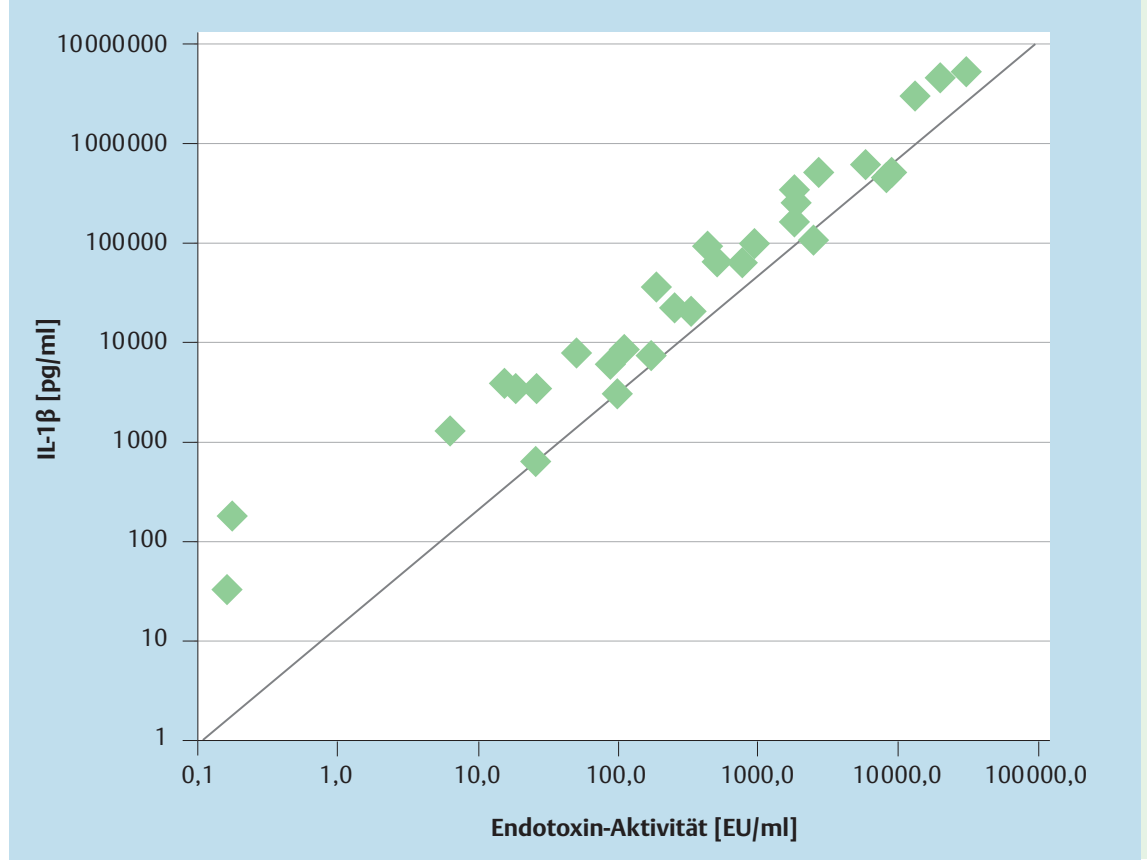

Abb. 4 Pyrogene Aktivität (IL-1ß-Freisetzung) und Endotoxin-Aktivität (LAL-Test) von 30 Staubfilterextrakten. Die Korrelation der Testergebnisse beträgt $r^{2}=0,9$ (Pearson). Zwei Proben, die im LAL-

Test an der unteren Nachweisgrenze liegen, zeigen im VBT deutliche Aktivität.

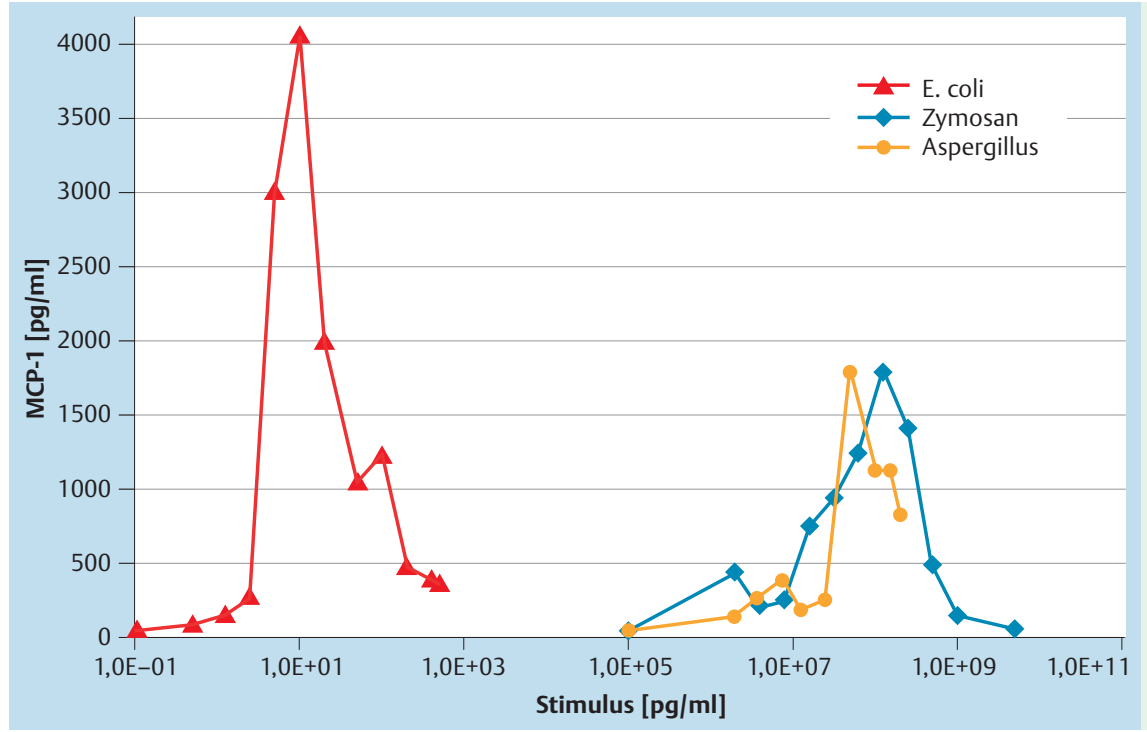

Abb. 5 MCP-1-Freisetzung von kryo-konserviertem Blut nach Stimulation mit E. coli-Endotoxin, Aspergillus versicolor-Extrakt und Zymosan. Während E. coli im Pikogrammbereich zu einer Antwort führt, werden von Aspergillus und Zymosan mg-Mengen zur Stimulation benötigt.

zentration von 10 bis $100 \mathrm{pg} / \mathrm{ml}$ für $E$. coli-Endotoxin und 1,2 bis $250 \mu \mathrm{g} / \mathrm{ml}$ für Zymosan oder A. versicolor-Extrakt bewirkten eine MCP-1-Antwort. Die maximale MCP-1-Menge lag für Zymosan und $A$. versicolor bei $1800 \mathrm{pg} / \mathrm{ml}$. Diese Menge des Chemokins wurde durch $50 \mu \mathrm{g} / \mathrm{ml} A$. versicolor bzw.125 $\mu \mathrm{g} / \mathrm{ml}$ Zymosan induziert. Eine vergleichbare MCP-1-Freisetzung erhält man nach Stimulation mit $20 \mathrm{pg} / \mathrm{ml}$ E. coli-Endotoxin. Die MCP-1-Freisetzung spricht also um den Faktor 1:1000000 sensitiver auf Endotoxin im Vergleich zu Pilzkomponenten an.

\section{Diskussion}

$\nabla$

Die vorliegende Studie validiert den Einsatz des VBT zur Beschreibung von Staubextrakten. Unsere Ergebnisse zeigen, dass die Freisetzung von IL-1 $\beta$ und MCP-1 aus kryo-konserviertem Blut geeignete Parameter sind, um die pyrogene Aktivität von arbeitsplatzrelevanten Proben zu bestimmen.
Der Einsatz von kryo-konserviertem statt frischem Vollblut erlaubt es, unterschiedliche Staubextrakte mit der gleichen Blutcharge zu charakterisieren und damit eine vergleichende Einschätzung vorzunehmen.

Der VBT kommt unter anderem in der Diagnostik gramnegativer Infektionen zum Einsatz [17]. Im Übrigen wird er vor allem genutzt, um die Kontamination von Medikamenten mit pyrogenen Substanzen zu überprüfen $[18,19]$.

Zur Charakterisierung von Arbeitsplätzen liegen bisher überwiegend Ergebnisse des VBT mit frischem Blut vor. Zucker et al. [20] beschrieben die IL-1 $\beta$-Freisetzung von Blut zehn nicht exponierter Spender, stimuliert mit Proben einer Entenzuchtanlage. Die Autoren fanden eine gute Korrelation zwischen LAL-Testergebnissen und IL-1 $\beta$-Freisetzung (Spearman $r=0,9$ ). Diese Ergebnisse bestätigen sich in unseren Untersuchungen. Allerdings ist zu bedenken, dass die Korrelation der beiden Tests von der Art der Probe abhängt: Gramnegative Bakterien sorgen für eine hohe Korrelation, da ihre Endotoxine sowohl im LAL-Test als auch im 


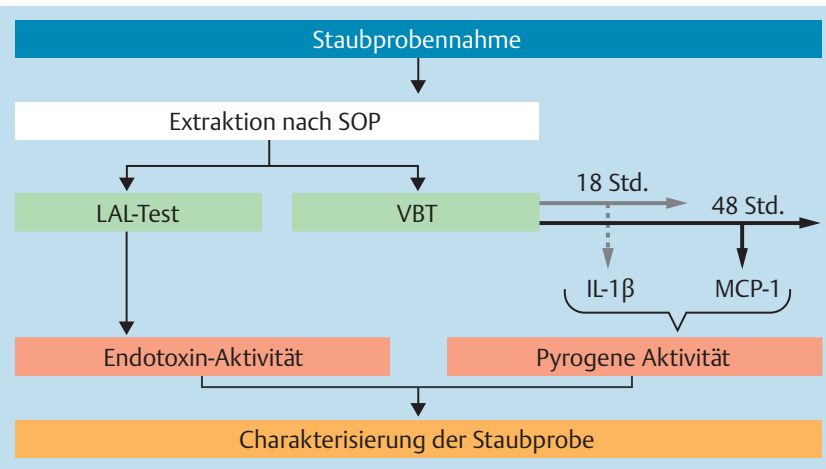

Abb. 6 Schematische Darstellung eines möglichen Ablaufplans zur Analyse von Staubfilterextrakten. SOP = standard operation procedure; VBT = Vollbluttest; LAL-Test $=$ Limulus-Amöbocyten-Lysat-Test; IL-1 $\beta=$ Interleukin-1 $\beta$; MCP-1 = monocyte chemotactic protein 1 .

VBT nachgewiesen werden können. Sind die Staubextrakte dagegen reich an grampositiven Bakterien, Pilzen und anderen mikrobiologischen Komponenten mit hoher pyrogener Aktivität, werden diese im LAL-Test nicht erfasst.

Um Staubproben zu charakterisieren, ist eine mehrstufige Analyse von Proben mit unterschiedlichen Testverfahren deshalb empfehlenswert (siehe $\bullet$ Abb. 6).

Wird der VBT mit frischem Blut einzelner Spender durchgeführt, lassen sich vor allem Informationen zur individuellen Suszeptibilität gegenüber Bioaerosol-bedingten Entzündungsreaktionen ableiten [21]. Smit et al. [22] konnten zeigen, dass sich eine hohe berufliche Endotoxin-Exposition auch im VBT widerspiegelt: Für die Gruppe der High Responder bestand ein signifikanter DosisWirkungs-Zusammenhang zwischen beruflicher Endotoxin-Exposition und respiratorischen Symptomen. Als High Responder wurden Personen bezeichnet, deren Blut nach In-vitro-Stimulation mit LPS im Mittel mehr Zytokine freisetzte als das Blut der Vergleichsgruppe (12 Personen).

Wenn frisches humanes Blut eingesetzt wird, ist die gemessene pyrogene Aktivität ein Maß für die individuelle biologische Reaktion auf einen gegebenen Stimulus (Beanspruchungsparameter). Genetische und umweltbedingte Faktoren spielen dabei ebenso eine Rolle wie intraindividuelle Veränderungen, z.B. in Abhängigkeit vom zirkadianen Rhythmus [23].

Setzt man dagegen kryo-konserviertes humanes Blut ein, erlaubt die gemessene pyrogene Aktivität eine Aussage über den Stimulus, also z.B. die Staubprobe (Belastungsparameter). Zusätzlich umgeht man mit kryo-konserviertem Blut das Problem, das Frischblut nur in begrenzter Menge zur Verfügung steht. Das Kryo-Blut stellt eine Mischung des Blutes verschiedener Spender dar [24,25].

Die IL-1 $\beta$-Messung wird beim Einsatz des VBT bevorzugt eingesetzt [12,18-20]. Die Messung weiterer Parameter, wie z.B. MCP-1, kann je nach Fragestellung integriert werden. Damit bietet der VBT flexible Möglichkeiten, neben Einzelsubstanzen auch arbeitsplatzbezogene Staubproben zu untersuchen. In unseren Untersuchungen zeigte die MCP-1-Freisetzung die geringste Korrelation zu den LAL-Test-Ergebnissen im Vergleich zu den anderen Zytokinen (IL-1 $\beta$, IL-6, IL-8, TNF- $\alpha$ ) [2]. Das könnte ein Hinweis darauf sein, dass in diesem Testverfahren Nicht-EndotoxinKomponenten, wie z.B. Pilzbestandteile, die MCP-1-Freisetzung induzieren. MCP-1 (monocyte chemoattractant protein) ist ein CC-Chemokin, das vor allem auf Monozyten wirkt und außerdem als Regulator in verschiedenen entzündlichen Krankheiten beim
Menschen eine Rolle spielt. Dass Pilzsporen eine MCP-1-Freisetzung induzieren können, wurde z.B. für die Sporen von Aspergillus fumigatus mit Zelllinien von Mäusen nachgewiesen [26]. Unsere Daten zeigen, dass Zymosan und $A$. versicolor-Extrakt eine MCP-1-Antwort im VBT auslösen können. Da der A. versicolor-Extrakt keine IL-1 $\beta$-Freisetzung induzierte, stellt die MCP-1-Messung eine Möglichkeit dar, um immunologisch aktive Komponenten nachzuweisen, die eine andere Signalkaskade anstoßen. Denkbar ist z.B., dass Pilzkomponenten, die via TLR-2 und Dectin-1 erkannt werden, eine andere Zytokinkaskade auslösen als Bakterienbestandteile, die im Wesentlichen mit TLR-2 und TLR-4 reagieren.

A. versicolor gehört neben Penicillium chrysogenum und Stachybotrys chartarum zu den häufigsten Arten, die in Innenräumen mit Feuchteschäden gefunden werden $[27,28]$. Aspergillus-Spezies sind generell die häufigsten Pilze, mit denen Menschen konfrontiert sind und die in organischen Stäuben vorkommen. Sie können eine Vielzahl von Krankheiten auslösen, obwohl keinesfalls jede Exposition gegenüber diesen Pilzen zu Beschwerden führt [29].

Insgesamt zeigen unsere Ergebnisse, dass der VBT ein sinnvolles Verfahren ist, um die proinflammatorische Antwort von Staubfilterproben und ihrer Einzelkomponenten zu beschreiben. Kryokonserviertes Blut ist geeignet, um Proben hinsichtlich ihrer pyrogenen Aktivität zu quantifizieren. Im Gegensatz zu Frischblut ermöglicht es, Belastungen an verschiedenen Arbeitsplätzen, unabhängig von der individuellen Beanspruchung exponierter Personen, zu beschreiben.

\section{Interessenkonflikt}

$\nabla$

Die Autoren geben an, dass kein Interessenkonflikt besteht.

\section{Literatur}

1 Liebers V, Brüning T, Raulf-Heimsoth M. Occupational endotoxin-exposure and possible health effects on humans. Am J Ind Med 2006; 49: 474-491

2 Liebers V, Stubel H, Düser M et al. Standardization of whole blood assay for determination of pyrogenic activity in organic dust samples. Int J Hyg Environ Health 2009; 212: 547-556

3 Rylander R. Endotoxin and occupational airway disease. Curr Opin Allergy Clin Immunol 2006; 6: 62-66

4 Sigsgaard T, Bonefeld-Jørgensen EC, Hoffmann HJ et al. Microbial cell wall agents as an occupational hazard. Toxicol Appl Pharmacol 2005; 207: 310 - 319

5 Dennehy KM, Brown GD. The role of the $\beta$-glucan receptor Dectin-1 in control of fungal infection. J Leukoc Biol 2007; 82: 253-258

6 Novak N, Bieber T, Peng WM. The immunoglobulin E-Toll-like receptor network. Int Arch Allergy Immunol 2010; 151: 1 - 7

7 Mutius E von, Braun-Fahrlander C, Schierl R et al. Exposure to endotoxin or other bacterial components might protect against the development of atopy. Clin Exp Allergy 2000; 30: 1230-1234

8 Douwes J, Travier N, Huang Ket al. Lifelong farm exposure may strongly reduce the risk of asthma in adults. Allergy 2007; 62: 1158-1165

9 Mastrangelo $G$, Fadda E, Rylander $R$ et al. Lung and other cancer site mortality in a cohort of Italian cotton mill workers. Occup Environ Med 2008; 65: 697-700

10 Singh J, Schwartz DA. Endotoxin and the lung: Insight into the host-environment interaction. J Allergy Clin Immunol 2005; 115: 330-333

11 Chun DT, Bartlett $K$, Gordon T et al. History and results of the two interlaboratory round robin endotoxin assay studies on cotton dust. Am J Ind Med 2006; 49: 301 - 306

12 Liebers V, Raulf-Heimsoth M, Linsel G et al. Evaluation of quantification methods of occupational endotoxin-exposure. J Tox Environ Health A 2007; 70: $1798-1805$ 
13 Spaan S, Heederik DJ, Thorne PS. Optimization of airborne endotoxin exposure assessment: effects of filter type, transport conditions, extraction solutions and storage of samples and extracts. Appl Environ Microbiol 2007; 73: 6134-6143

14 Brandenburg K, Howe J, Gutsman $T$ et al. The expression of endotoxic activity in the limulus test as compared to cytokine production in immune cells. Curr Med Chem 2009; 16: 2653 - 2660

15 Kindinger I, Daneshian M, Baur $H$ et al. A new method to measure airborne pyrogens based on human whole blood cytokine response. J Immunol Meth 2005; 298: 143-153

16 Thrasher JD, Crawley S. The biocontaminants and complexity of damp indoor spaces: more than what meets the eyes. Toxicol Ind Health 2009; 25: $583-615$

17 Stoll LL, Denning GM, Weintraub NL. Potential role of endotoxin as a proinflammatory mediator of atherosclerosis. Arterioscler Thromb Vasc Biol 2004; 24: 2227-2236

18 Daneshian M, Guenther A, Wendel A et al. In vitro pyrogen test for toxic or immunomodulatory drugs. J Immunol Meth 2006; 313: 169-175

19 Hasiwa M, Kullmann K, Aulock S von et al. An in vitro pyrogen safety test for immune-stimulating components on surfaces. Biomaterials 2007; 28: $1367-1375$

20 Zucker B-A, Scharf P, Kersten C. Determination of the inflammatory potential of bioaerosols from a duck-fattening unit by using a limulus amebocyte lysate assay and human whole blood cytokine response. J Vet Med 2006; B53: 176-180
21 Wouters I, Douwes J, Zhorne P et al. Inter- and intraindividual variation of endotoxin- and $\beta(1 \rightarrow 3)$-glucan-induced cytokine responses in a whole blood assay. Tox Industrial Health 2002; 18: 15 - 27

22 Smit LA, Heederik D, Doekes $G$ et al. Ex vivo cytokine release reflects sensitivity to occupational endotoxin exposure. Eur Respir J 2009; 34: $795-802$

23 Böttcher MF, Hmani-Aifa M, Lindström A et al. A TLR4 polymorphism is associated with asthma and reduced lipopolysaccharide-induced interleukin-12(p70) responses in Swedish children. J Allergy Clin Immunol 2004; 114: 561 - 567

24 Schindler S, Asmus S, von Aulock S et al. Cryopreservation of human whole blood for pyrogenicity testing. J Immunol Meth 2004; 294: 89 100

25 Schindler S, Spreitzer I, Löschner B et al. International validation of pyrogen tests based on cryopreserved human primary blood cells. J Immunol Methods 2006; 316: $42-51$

26 Pylkkänen L, Gullstén H, Majuri ML et al. Exposure to Aspergillus fumigatus spores induces chemokine expression in mouse macrophages. Toxicology 2004; 200: 255-263

27 Gravesen S, Nielsen PA, Iversen $R$ et al. Microfungal contamination of damp buildings - examples of risk constructions and risk materials. Environ Health Perspect 1999; 107: 505-508

28 Bellanger AP, Reboux G, Roussel S et al. Indoor fungal contamination of moisture-damaged and allergic patient housing analysed using realtime PCR. Lett Appl Microbiol 2009; 49: 260 - 266

29 Park SJ, Mehrad B. Innate immunity to Aspergillus species. Clin Microbiol Rev 2009; 22: $535-551$ 